\title{
Lightweight optimization design of automotive aluminum alloy front
}

\section{bumper anti-collision beam}

\author{
Deng-feng Wang, Chuan-qing Wang
}

(State Key Laboratory of Automotive Simulation and Control, Jilin University, Changchun 130022 China)

caewdf@jlu.edu.cn, jilinchuanqing@foxmail.com

\begin{abstract}
Keywords: Lightweight optimization design, aluminum alloy anti-collision beam, morphing technology, crashworthiness, stiffness

Abstract. The design of aluminum alloy front bumper beam is based on steel bumper. The process is divided into two stages. The first stage is topology optimization, and the second stage is size optimization. Two thin-walled, hollow aluminum bumpers with reinforced ribs is obtained after topology optimization. Take the thickness of all surfaces and the distance of two reinforced ribs as design variables to conduct size optimization, while maintain the performances of crashworthiness and stiffness. After optimization two type anti-collision aluminum alloy beams are obtained. The comparison results show that the performances of two type anti-collision aluminum alloy beam are better than steel beam, and the mass is smaller. The performance of second type anti-collision beam is better than the first type, but the mass is bigger.
\end{abstract}

\section{Introduction}

Lightweight is an effective way to solve the problem of automobile fuel consumption and emission. One of important ways to achieve lightweight is developing aluminum parts [1]. The front bumper anti-collision beam is a typical frontal-crash safety part. It has a great influence on frontal crash. Wang guan, Zhou jia, etc. designed an aluminum alloy bumper anti-collision beam. After the performance analyzed and compared with steel anti-collision beam. The energy absorption ability is increased $45.6 \%$, while the mass is reduced at the same time [2]. SIMON P, BEGGS P D analyzed the positive column collision simulation with $16 \mathrm{~km} / \mathrm{h}$ of DP600 high strength steel and aluminum alloy bumper through LS-DYNA software. The results show that if the mass of two bumpers is equal, the crashworthiness performance of aluminum alloy bumper is better [3]. In the process of developing anti-collision beam with new material, topology optimization is important. Grujicic, Arakere, et al, get new optimal structure with polymer metal hybrid (PMH) material through topology optimization [4]. Structure optimization method of the dynamic characteristic includes two categories which are done based on surrogate model [5-6] and integrate software solvers [7-8]. The optimization based on surrogate model is used to solve the large scale optimization problems, which is efficient but the initial model is complex. The optimization of integrate software solvers is used to solve the small scale optimization problems, which is accuracy. In this paper, the topology optimization is done firstly, and then the size optimization is done using the method of integrate software solvers. After getting the optimized aluminum alloy anti-collision beam, the performances are compared with the steel anti-collision beam. 


\section{Topology optimization design of aluminum alloy anti-collision beam}

Before optimization, the stiffness and crashworthiness of steel anti-collision beam are done, which will be compared with aluminum alloy beam. The models for analysis of stiffness and crashworthiness are shown in Fig. 1 and Fig. 2 respectively. When analyze stiffness, extract 5KN force in the middle location of the outer side, and constrain all the freedom of the bracket. When analyze crashworthiness, the initial velocity is $5 \mathrm{KM} / \mathrm{h}$. After counterweight, the mass is $1301.03 \mathrm{~kg}$. This paper takes static displacement, energy absorption and Tompkins displacement as performance index.

According to the geometry size of the steel anti-collision beam, the topology space is determined as shown in Fig.3. Constrain all the freedom of the left side, and all the freedom of the right side except the translation of $Y$. This paper uses static force to equal dynamic force, and put uniform load on the outside of the anti-collision beam. The magnitude is $260 \mathrm{KN}$, which is the average force of the frontal crash analysis. Extract the extrusion in the molding direction in order to keep the cross section not changing.

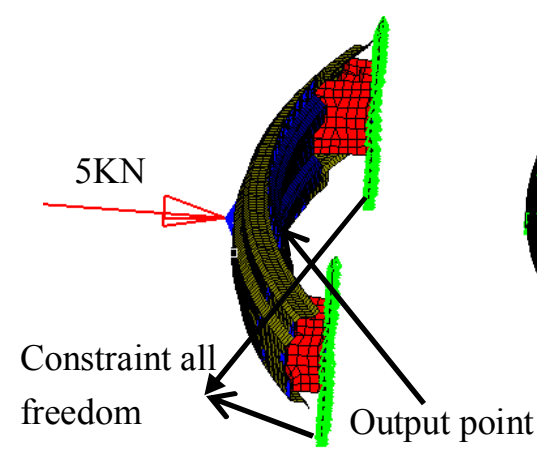

Fig.1 analyze of stiffness

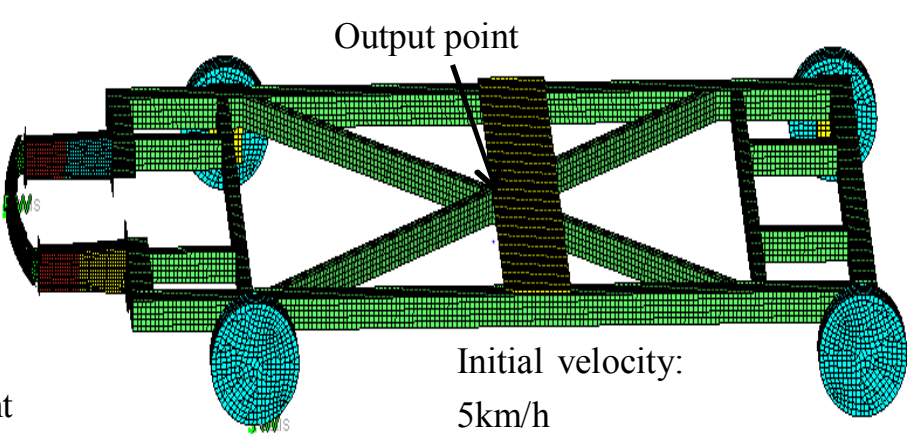

Fig.2 analyze of crashworthiness

The objective is making static strain energy minimum. Adjust the volume fraction; this paper gets two different type of cross section. The first type of anti-collision has one rib in the middle of the cross section when the volume fraction is 0.2 . The second type of anti-collision has two ribs which divide the whole section into three segments equally when the volume fraction is 0.3 . The first type cross section and the second type cross section are shown in Fig.4 (a) and Fig.4 (b).

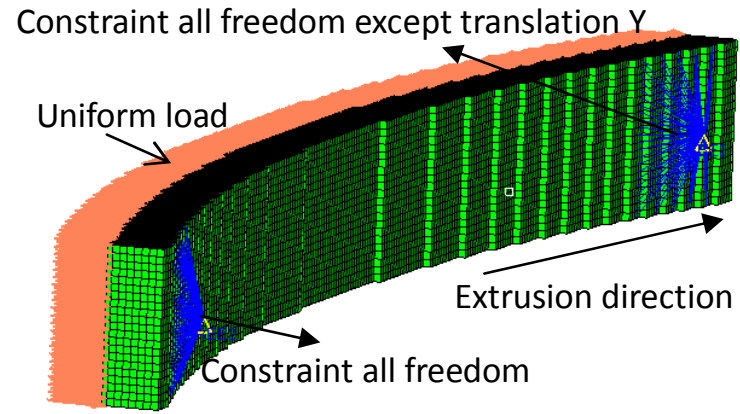

Fig.3 Topology space of aluminum alloy anti-collision beam

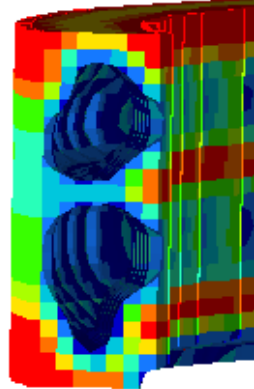

(a) first structure

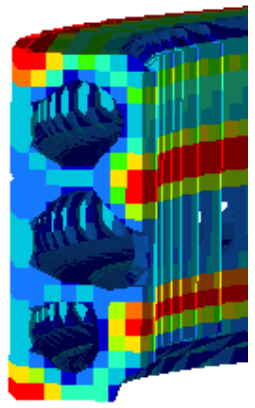

(b) second structure
Fig.4 the topology optimization structure

\section{Size optimization design of aluminum alloy anti-collision beam}

After topology optimization, size optimization is done for the two type cross sections. Considering the constraint of the assembling, shapes, et al, the first type only changes thickness of the structure, and the second type changes thickness of the structure and the distance of the two ribs. The thickness variable of the first type cross section is shown in Fig.5. The change of the second type 
cross section is shown in Fig.6. In Fig.5, $\delta 1, \delta 2$ are the thickness of the front and rear side of the anti-collision beam, $\delta 3$ is the thickness of the upper and lower side of the anti-collision beam, $\delta$ 4 is the thickness of the rib. In Fig.6, $\mathrm{L}$ is half distance of the two ribs. In the process of optimization, the thickness variable is discrete, the minimum interval is $0.1 \mathrm{~mm}$. The distance change of the two ribs is achieved through morphing technology. The objective of optimization is to minimize the mass of anti-collision beam and displacement of tompkins, while keeping the stiffness and crashworthness not being reduced. The expression of mathematical model are shown as follows:

Objective: $\quad\left\{\min (\operatorname{mass}), \operatorname{minv}_{\mathrm{lv}}(\mathrm{dis})\right\}$

Subject to: $\quad \mathrm{s}_{\mathrm{lv}}($ dis $) \leq \mathrm{s}_{\mathrm{st}}(\mathrm{dis})$;

$$
\mathrm{E}_{\mathrm{st}} \leq \mathrm{E}_{\mathrm{lv}}
$$

variable: $\quad 1.5 \leq \delta 1, \delta 2, \delta 3, \delta 4 \leq 5.0 ; \quad-10 \leq \mathrm{L} \leq 10$

Where, $\mathrm{s}_{\mathrm{lv}}(\mathrm{dis}), \mathrm{s}_{\mathrm{st}}(\mathrm{dis})$ are the tompkins displacement of aluminum alloy and steel anti-collision bumper respectively; $\mathrm{E}_{\mathrm{st}}, \mathrm{E}_{\mathrm{lv}}$ are the energy absorption of the aluminum alloy and steel anti-collision bumper respectively.

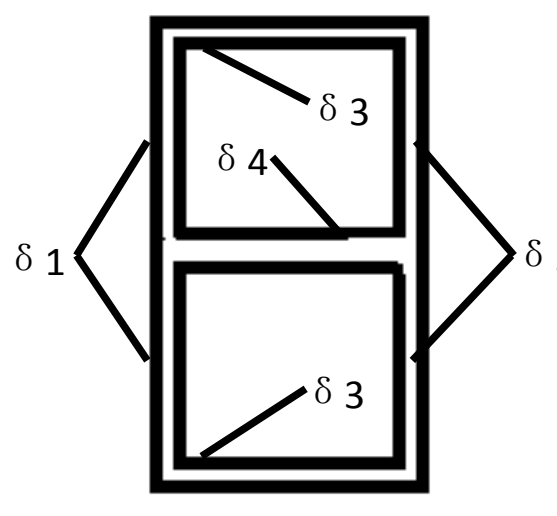

Fig.5 the variable of the first type

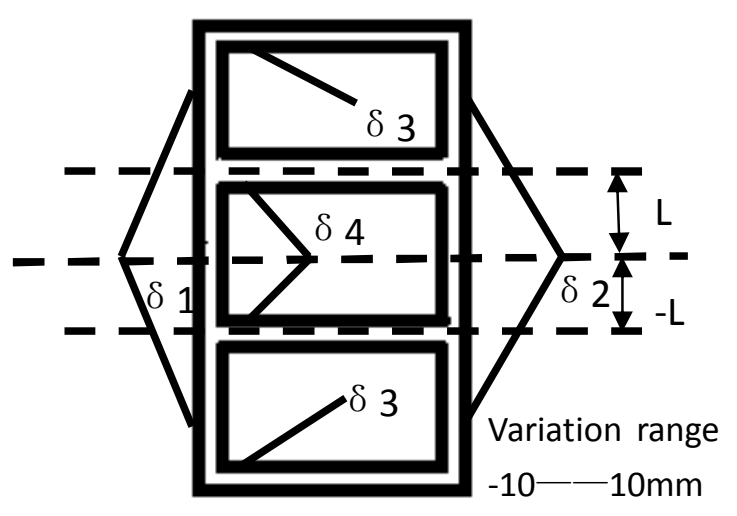

Fig.6 the variable of the second type

The size optimization is done through ISIGHT. The optimization algorithm is Non- Dominate Sorting Genetic Algorithm (NSGA- II ). The parameters of NSGA- II are as Tab.1.

Tab.1 The parameters of NSGA- II

\begin{tabular}{|c|c|c|c|c|c|}
\hline Population size & 20 & Crossover probability & 0.9 & mutation distribution index & 20.0 \\
\hline Number of generations & 30 & Crossover distribution index & 10.0 & & \\
\hline
\end{tabular}

Though the first type anti-collision beam doesn't have shape variable, the optimization process is the same as the second type.

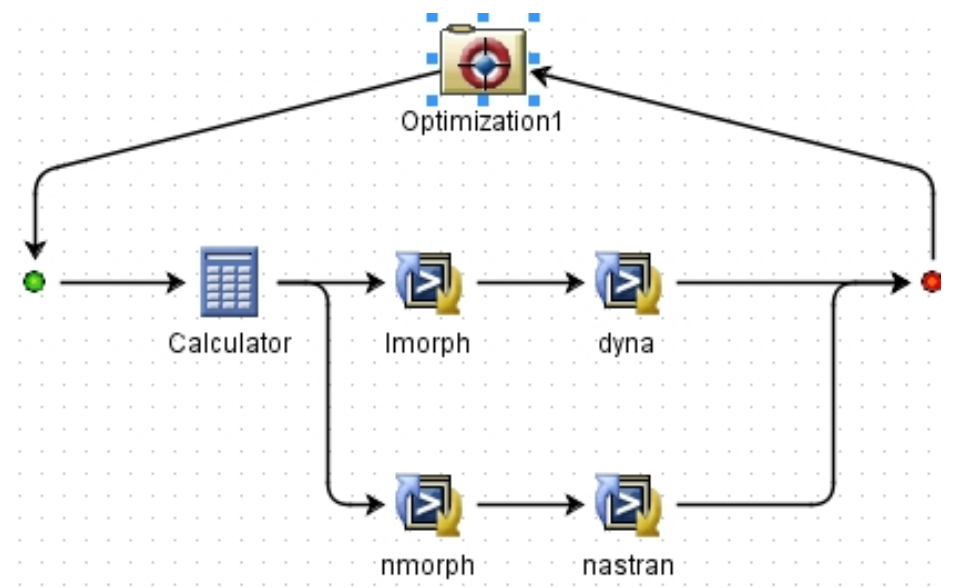

Fig. 7 the process of optimization 
The optimization process is shown in Fig.7. In Fig.7, the module of "dyna" and "Nastran" are solves for crashworthiness and stiffness analysis. The module of "lmorph" and "nmorph" are software of Meshworks to generate model for crashworthiness and stiffness. The module of "calculate" is to bring crashworthiness model into correspondence with stiffness model.

\section{Parameters comparison between aluminum alloy and steel anti-collision beam}

After optimization, this paper obtains the optimized structures and comparison with steel anti-collision beam. Fig. 2 is the thickness and the distance between the two ribs of the two type optimized anti-collision beam. Fig. 3 is the performances comparison of all anti-collision beams. As can be seen in Fig.3, the performances of the optimized two type aluminum alloy anti-collision beams are better, and the mass is smaller.

Tab.2 The structure of the second type optimized anti-collision beam

\begin{tabular}{|c|c|c|c|c|c|}
\hline Name & $\delta 1 / \mathrm{mm}$ & $\delta 2 / \mathrm{mm}$ & $\delta 3 / \mathrm{mm}$ & $\delta 4 / \mathrm{mm}$ & $2 \mathrm{~L} / \mathrm{mm}$ \\
\hline First type & 1.9 & 2.4 & 1.5 & 1.5 & 0 \\
\hline Second type & 1.5 & 2.9 & 4.8 & 1.5 & 17.2 \\
\hline
\end{tabular}

Tab.3 The performance comparison of all anti-collision beams

\begin{tabular}{|c|c|c|c|c|}
\hline & Mass/Kg & Energy absorption/J & $\begin{array}{c}\text { Static } \\
\text { displacement } / \mathrm{mm}\end{array}$ & Tompkins displacement/mm \\
\hline steel & 3.667 & 6316.82 & 40.48 & 482.3 \\
\hline First type & 2.134 & 7694.48 & 35.43 & 478.42 \\
\hline Second type & 2.976 & 7854.39 & 33.86 & 472.92 \\
\hline
\end{tabular}

\section{Conclusion}

Through the above profound analysis and optimization, following research results can be acquired:

(1) Two different type cross section anti-collision beams are obtained through topology optimization.

(2) For further research, take the thickness and shape as variables to conduct size optimization.

(3) The performances of two optimized aluminum alloy anti-collision beams and steel beam are analyzed and compared. The two type aluminum alloy anti-collision beams are better, and the mass is smaller. The performance of second type anti-collision beam is better than the first type, but the mass is bigger.

\section{Acknowledgements}

This research work was supported by Chongqing Basis and Leading-edge Research Program Projects (cstc2013jcyjC60001). The authors would like to express their appreciations for the above fund supports. 


\section{References}

[1] ZHU Ping, ZHANG Yu, GE Long, et al. Research on lightweight of auto-body material based on crashworthiness simulation [J]. Journal of Mechanical Engineering, 2005, 41(9): 207-211.

[2] WANG Guan, ZHOU Jia, LIU Zhi wen, et al. Lightweight design and crash performance analysis of automotive aluminum bumper [J]. The Chinese Journal of Nonferrous Metals, 2012, 22(1): 91-98.

[3] SIMON P, BEGGS P D. A numerical performance comparison of a dual-phase steel and aluminum alloy bumper bar system [J]. International Journal of Crashworthiness, 2010, 15(4): 425-442.

[4] M. Grujicic, G. Arakere, P. Pisu, B. etc. Application of Topology, Size and Shape Optimization Methods in Polymer Metal Hybrid Structural Lightweight Engineering [J], Multidiscipline Modeling in Materials and Structures, 2008, (4): 305-330.

[5] David Guirguis, Karim Hamza, Mohamed Aly, et al. Multi-objective topology optimization of multi-component continuum structures via a Kriging-interpolated level set approach [J]. Structural Multidiscipline Optimization, DOI 10.1007/s00158-014-1154-3.

[6] Chang-Chun Zhou, Guo-Fu Yin, Xiao-Bing Hu. Multi-objective optimization of material selection for sustainable products: Artificial neural networks and genetic algorithm approach [J]. Materials and Design, 2009, 30: 1209-1215.

[7] XU Zhong ming, XU Xiao fei, WAN Xin ming, et al. Structure Optimal Design of Aluminum Alloy Bumper Anti-collision Beam [J]. Journal of Mechanical Engineering, 2013, 49(8): 136-142.

[8] ZHANG Z H, LIU S T, TANG Z L. Design optimization of cross-sectional configuration of rib-reinforced thin-walled beam [J]. Thin-walled Structure, 2009, 47: 868-878. 